\title{
Hidrólise-hidrogenação catalítica do óleo de soja visando à produção de ácidos graxos livres (AGL)
}

\author{
C. A. B. CRISÓSTOMO ${ }^{1}$, K. M. SOUZA ${ }^{2}$, T. S. S. ALMEIDA ${ }^{2}$, R R. SOARES ${ }^{1,2}$ \\ ${ }^{1}$ Universidade Federal de Uberlândia, Departamento de Biocombustíveis \\ ${ }^{2}$ Universidade Federal de Uberlândia, Departamento de Engenharia Química \\ E-mail para contato: camilobrandeq@gmail.com/ tata_zinha_105@hotmail.com
}

\begin{abstract}
RESUMO - Recentemente foi verificado que a solução aquosa do glicerol, formada após a hidrólise de óleos utilizando catalisadores heterogêneos, pode sofrer a reação de reforma em fase líquida levando à formação de $\mathrm{H}_{2}$ e $\mathrm{CO}_{2} . \mathrm{O} \mathrm{H}_{2}$ pode ser usado na reação de hidrogenação dos ácidos graxos livres insaturados formados, permitindo a obtenção de um ácido graxo específico de maior valor agregado. O presente trabalho busca selecionar um catalisador ativo para a reação de hidrólise, utilizando o óleo de soja não refinado como reagente, e para a reação subsequente, a hidrogenação dos ácidos graxos. Os testes catalíticos foram realizados com catalisadores $\mathrm{NiO}$, calcinados a diferentes temperaturas, e Ni-Sn (razão molar 1:1), todos suportados em $\gamma$-alumina. Para a reação, foi utilizado um reator batelada de $500 \mathrm{~mL}$. Os produtos foram obtidos após 4 horas de reação. A composiçao de ácidos graxos livres no produto foi determinada por cromatografia gasosa. Os resultados mostraram que os catalisadores foram ativos e estáveis para a reação de hidrólise. Foi verificado em todas as reações que ocorreu a conversão de glicerol por APR.
\end{abstract}

\section{I - INTRODUÇÃO}

Recentemente foi verificado que a solução aquosa do glicerol, formada após a hidrólise de óleos utilizando catalisadores heterogêneos, pode sofrer a reação de reforma em fase líquida (APR - Aqueous Phase Reforming) levando a formação de hidrogênio e dióxido de carbono. Este hidrogênio pode atuar na reação de hidrogenação dos ácidos graxos, formando ácidos graxos saturados, os quais são de alta relevância para indústrias alimentícias e oleoquímicas. Dessa forma, a hidrogenação além de melhorar a qualidade dos AGL, permite a obtenção de um ácido graxo específico de maior valor agregado (Diaz et al., 2011). Catalisadores a base de platina, paládio e níquel são promissores para este tipo de reação. Catalisadores de níquel podem ser uma alternativa atrativa para este tipo de processo devido ao menor custo.

O processo de hidrólise e hidrogenação simultânea envolve basicamente três etapas, as quais são: hidrólise, reforma em fase liquida do glicerol e a hidrogenação. A Figura 2 a seguir mostra como ocorre a combinação destes processos.

Neste sentido, o presente trabalho busca selecionar um catalisador ativo para a reação de hidrólise de óleos vegetais e para a reação subsequente, a hidrogenação dos ácidos graxos a 
partir do $\mathrm{H}_{2}$ obtido do glicerol. Os testes catalíticos foram realizados com óleo de soja não refinado, catalisadores $15 \% \mathrm{NiO} / \gamma-\mathrm{Al}_{2} \mathrm{O}_{3}$, calcinados a diferentes temperaturas; e um catalisador bi metálico $\mathrm{Ni}-\mathrm{Sn} / \gamma-\mathrm{Al}_{2} \mathrm{O}_{3}$, razão molar de $\mathrm{Ni} / \mathrm{Sn}$ de $1: 1$.

Figura 1 - Processo de hidrólise e hidrogenação simultânea (Diaz et al., 2011)

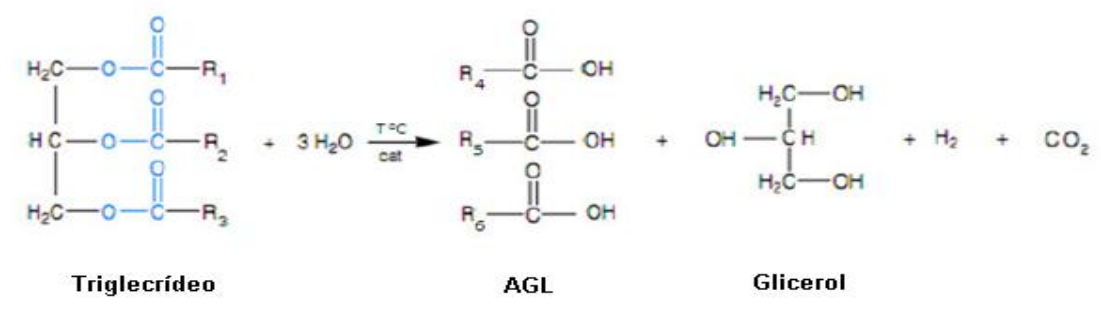

\section{II - MATERIAIS E MÉTODOS}

\section{1 - Preparo e caracterização dos catalisadores}

Foram preparados catalisadores monometálicos contendo $15 \%$ em peso de $\mathrm{NiO}$, suportados em $\gamma-\mathrm{Al}_{2} \mathrm{O}_{3}$ (G-250-Basf), previamente calcinada em mufla a $500^{\circ} \mathrm{C}$ por 4 horas. Os catalisadores foram preparados pelo método da impregnação seca do suporte com uma solução aquosa de $\mathrm{Ni}\left(\mathrm{NO}_{3}\right)_{2} \cdot 6 \mathrm{H}_{2} \mathrm{O}$. Posteriormente, realizou-se a etapa de secagem a $100^{\circ} \mathrm{C}$, por 24 horas em estufa. Os catalisadores obtidos foram então calcinados a diferentes temperaturas, sendo estas: 300 e $500{ }^{\circ} \mathrm{C}$, por 4 horas. Estes foram então nomeados como NiO300 e NiO-500. O catalisador bi metálico foi preparado a partir da impregnação seca do catalisador NiO-seco, com uma solução aquosa de $\mathrm{SnCl}_{2}$, de forma a obter a razão molar de $\mathrm{Ni}-\mathrm{Sn}$ desejada $(1: 1)$ e foram em seguida calcinados a $300^{\circ} \mathrm{C}$, por 4 horas. $\mathrm{O}$ teor em massa obtido para o catalisador Ni-Sn foi de $17,2 \%$ de Sn e $8,6 \%$ de Ni.

A caracterização foi feita através de análises de Redução a Temperatura Programada (TPR) e Difração de Raios-X (DRX). As análises de TPR foram realizadas em uma unidade multipropósito acoplada a um espectrômetro de massas Balzers Omnistar. As análises de DRX foram feitas em um equipamento Shimadzu XRD-600, utilizando radiação CuK $\alpha$ $\left(1,5406 \AA\right.$ ). Os difratogramas foram obtidos nas seguintes condições: varredura entre $2 \theta=20^{\circ}$ e $80^{\circ}$, com um passo de $0,05^{\circ}$ e um tempo de contagem de 1 segundo por passo.

\subsection{Testes catalíticos}

Para a realização das reações de hidrólise foi utilizado um reator batelada de $500 \mathrm{~mL}$ (Snipe-Tite), equipado com um sistema de agitação e controlador de aquecimento. As condições reacionais estudadas foram: temperatura de $250{ }^{\circ} \mathrm{C}$, pressão inicial em torno de 25 bar, agitação entre 800-900 rpm, tempo de reação de 2 horas, razão mássica de óleo/agua de $1: 1$ e $10 \%(\mathrm{~m} / \mathrm{m})$ de catalisador em relação ao óleo. Dessa forma, a mistura reacional consistiu em 100 g óleo de soja não comercial fornecido pela CARAMURU, $100 \mathrm{~g}$ de água e $10 \mathrm{~g}$ de catalisador. 
Foi realizado um teste na ausência de catalisador ("teste em branco"), nas mesmas condições reacionais acima mencionadas, para servir como parâmetro de comparação no estudo da reação.

\subsection{Análises dos produtos da reação}

A fase oleosa obtida após a separação foi analisada por cromatografia gasosa utilizando um cromatógrafo Agilent, modelo 7890A, equipado com autoamostrador ALS 7650A, injetor Split/Splitless e detector de ionização de chama (FID). O teor de AGL foi determinado mediante metodologia EN 14103 adaptada (Pinese et al., 2013. As concentrações de AGL foram determinadas através do fator de resposta (fr) obtido de uma curva de calibração para cada padrão, como mostra a Equação 1. As amostras dos produtos foram diluídas a cerca de $10 \%(\mathrm{~m} / \mathrm{m})$ em heptano para análise no cromatógrafo.

$$
\%(\boldsymbol{m} / \boldsymbol{m})_{A G L}=\boldsymbol{f r} * \hat{A} \boldsymbol{r e a} \text { análise } * \text { Diluição } * 100
$$

A fase aquosa também foi analisada, com o intuito de quantificar o teor de glicerol possivelmente presente após a hidrólise. As análises foram feitas em um cromatógrafo Shimadzu, modelo GC-2010 equipado com, injetor Split/Splitless, detector de ionização de chama (FID).

\section{RESULTADOS E DISCUSSÃO}

\subsection{Caracterização dos catalisadores}

A Figura 2 a seguir mostra os resultados de TPR obtidos, indicando as temperaturas máximas de redução.

A presença de mais de um pico de consumo máximo de hidrogênio, em catalisadores onde o suporte é a alumina, ocorre devido ao fato de uma parte do metal estar mais exposta, ou seja, mais acessível a essa redução e o restante do metal apresentar uma maior interação com o suporte, possuindo menor acessibilidade e consequentemente reduzindo a uma temperatura mais elevada. Picos em temperaturas menores que $400^{\circ} \mathrm{C}$, como observado no $\mathrm{NiO}-300$, podem ser atribuídos à decomposição de nitratos residuais. Picos entre 400 e $700^{\circ} \mathrm{C}$, observado em todos os catalisadores calcinados, podem ser relacionados à redução de espécies de óxido de níquel presentes na fase "bulk", à redução do $\mathrm{NiO}$ com menor interação com o suporte, ou, ainda, à redução de espécies $\mathrm{Ni}^{2+}$, superficiais e altamente dispersas, a $\mathrm{Ni}$ metálico. Já picos próximos a $900^{\circ} \mathrm{C}$ correspondem a uma estrutura estável denominada aluminato de níquel (Scheffer et al., 1989).

Para os catalisadores $\mathrm{NiO} / \mathrm{Al}_{2} \mathrm{O}_{3}$, observou-se que o aumento da temperatura de calcinação promoveu o aumento na temperatura de redução. Quando comparado ao catalisador NiO-300, verifica-se que a adição de $\mathrm{Sn}$ também faz com que a redução se inicie a temperaturas mais elevadas, com o desaparecimento do pico redução a $356{ }^{\circ} \mathrm{C}$. 
Figura 2 - Perfis de TPR dos catalisadores testados

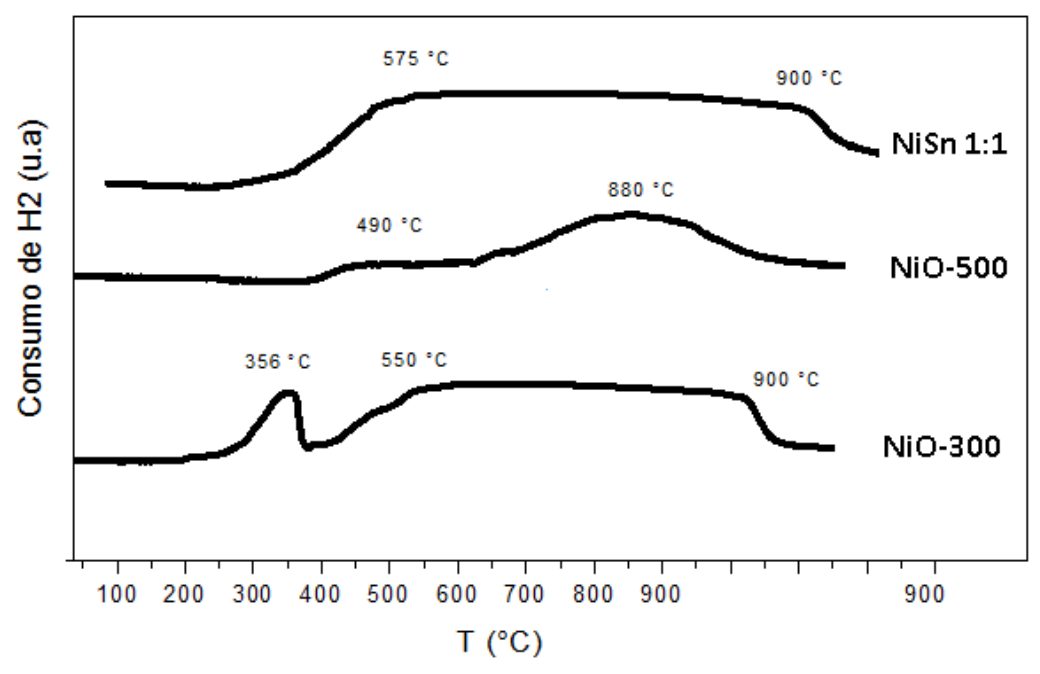

A Figura 4 mostra os resultados de DRX dos catalisadores, da alumina utilizada e do $\mathrm{NiO}$ obtido pela calcinação do $\mathrm{Ni}\left(\mathrm{NO}_{3}\right)_{2} \cdot 6 \mathrm{H}_{2} \mathrm{O}$ a $400^{\circ} \mathrm{C}$ por 3 horas.

Figura 3- Difratogramas dos catalisadores testados

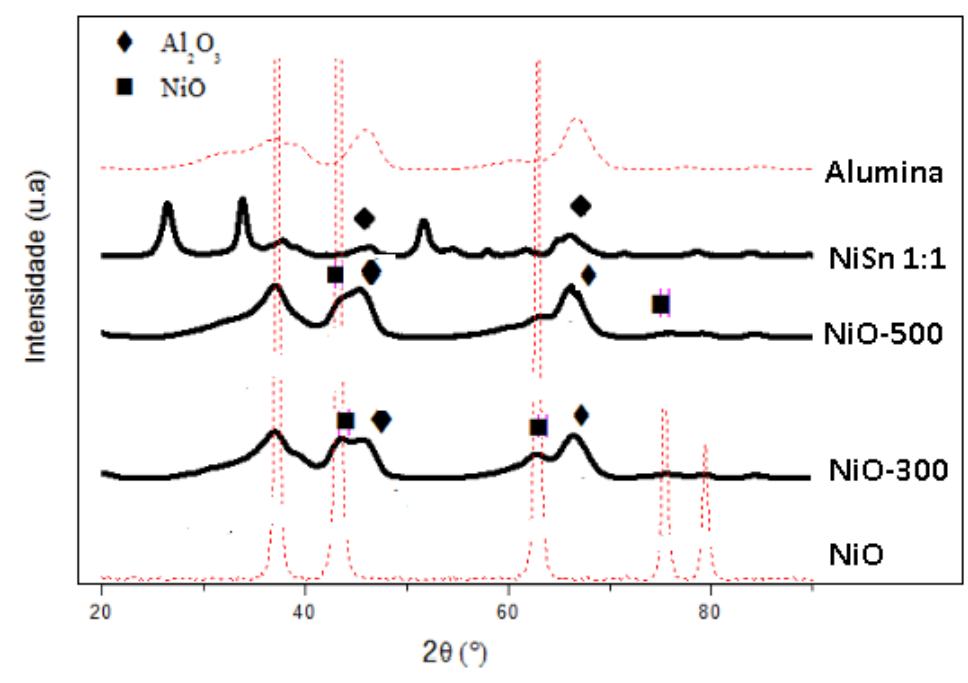

Os resultados mostram que a calcinação promoveu o aparecimento da espécie $\mathrm{NiO}$ nos catalisadores, visualizada na posição $2 \theta=43,2^{\circ}$. Segundo a ficha JCPDS este pico é característico da fase $\mathrm{NiO}$ cristalina (bunsenite de estrutura cúbica e grupo espacial Fm3m). O difratograma do catalisador não calcinado não apresentou a fase NiO. No catalisador bi metálico não foi verificado picos de difração que possam ser atribuídos ao $\mathrm{NiO}$.

\subsection{Testes catalíticos}

O objetivo na análise dos produtos da reação foi quantificar a porcentagem molar de ácidos graxos livres formados e a conversão de Triglicerídeos (TG). A análise por cromatografia gasosa do óleo de soja usado como reagente mostrou uma quantidade mínima 
de AGL presentes. Todos os catalisadores se mostraram ativos para a reação de hidrólise, uma vez que no teste em branco foi verificada uma conversão de TG menor que $1 \%$, ou seja, não houve produção de AGL nas condições reacionais estudadas $\left(250^{\circ} \mathrm{C}\right.$ e 48 bar), na ausência de catalisador. A figura 4 mostra a conversão de TG:

Figura 4 - Conversão de Triglicerídeos (TG)

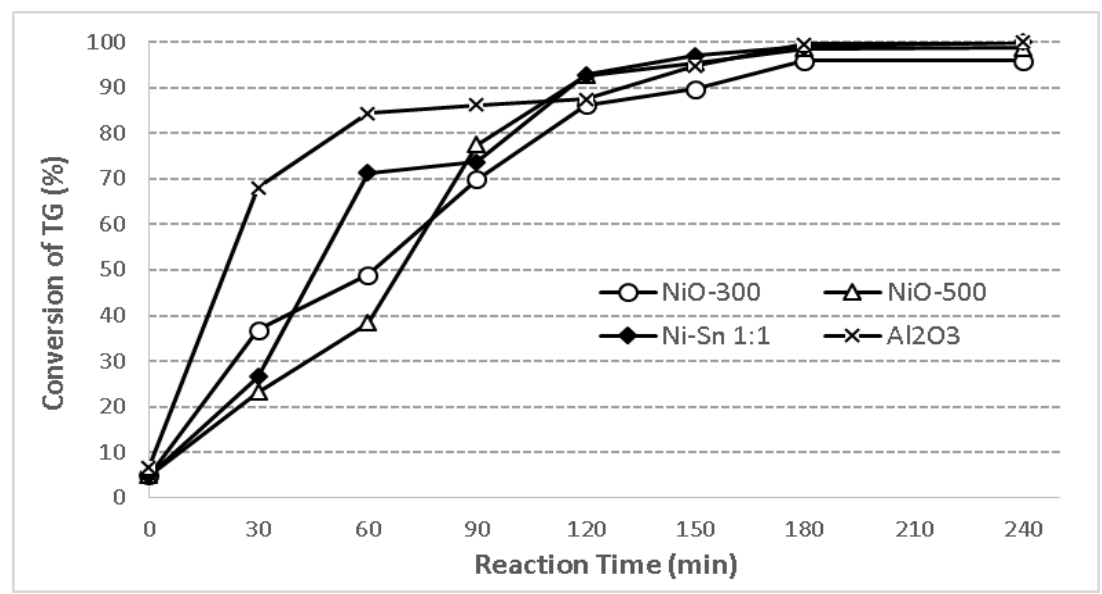

Verificou-se que a adição de Sn aumentou a conversão de TG para quase $100 \%$. A conversão foi alta já em 2 horas de reação, permanecendo assim até o final. A alumina também alta conversão de TG, sendo esta em torno de $100 \%$. Uma explicação para este resultado é que, teoricamente, a hidrólise ocorre em sítios ácidos e todos os catalisadores testados apresentam o mesmo teor de $\mathrm{Al}_{2} \mathrm{O} 3$ (85 \%), o sítio ácido no caso. Conforme mostrado em resultados de caracterização, a calcinação pode ter promovido uma interação entre a fase óxida e o suporte, reduzindo, em parte, a atividade do sítio ácido. A alumina apresentou o maior rendimento em AGL totais, sendo este de $71 \%$. Os catalisadores metálicos apresentaram rendimentos próximos, em torno de $35 \%$, sendo que este aumentou consideravelmente após 2 horas. O rendimento em AGL totais aumentou consideravelmente com a adição de $\mathrm{Sn}$, elevando-se até $58 \%$. A adição de $\mathrm{Sn}$ aumentou o rendimento em ácido esteárico, sendo que os rendimentos obtidos foram: $13 \%$ (Ni-Sn), $7 \%$ (NiO-300), $6 \%$ (NiO$500)$ e $3,2 \%$ (Alumina).

Os resultados da tabela 1 referem-se ao produto isento de $\mathrm{MG}+\mathrm{DG}$ (produtos intermediários) o qual pode ser obtido através de uma destilação, devido a grande diferença de volatilidade entre os ácidos graxos e os glicerídeos. O rendimento de MG e DG foi bastante reduzido no teste com o catalisador Ni-Sn, o que levou, consequentemente, a uma maior produção de AGL. Um maior tempo de reação também pode levar a conversão completa destes produtos intermediários em AGL.

Observou-se em todos os testes que a seletividade para o ácido esteárico (C18:0) aumentou e a do ácido linolênico e oleico diminuíram, quando comparada a uma reação com 100\% de conversão dos glicerídeos (Tabela 1), sem ocorrência de hidrogenação. Tal fato mostra que ocorreu a hidrogenação dos ácidos insaturados obtidos. Utilizando o catalisador NiO-300, a seletividade para o ácido esteárico diminui ao longo da reação. Já utilizando o catalisador com Sn, a seletividade para o C 18:0 aumenta com o tempo, podendo aumentar 
ainda mais com um maior tempo de reação. A alumina apresentou elevada conversão de TG, no entanto os resultados da Tabela 1 mostram que não ocorreu a hidrogenação ao se utilizar este catalisador.

A Tabela 1 mostra a seletividade em AGL do produto obtido.

Tabela 1 - Seletividade em AGL do produto obtido

\begin{tabular}{ccccc}
\hline & C 16:0 & C 18:0 & C 18:1 & $\underline{\text { C 18:2 }}$ \\
\hline S 100\% & 13,99 & 4,34 & 24,86 & 58,36 \\
\hline S/c (branco) & $<0,01$ & $<0,01$ & $<0,01$ & $<0,01$ \\
\hline Y-Al2O3 & 13,19 & 4,48 & 25,77 & 56,56 \\
\hline NiO-300 & 16,35 & 20,90 & 17,33 & 45,42 \\
\hline NiO-500 & 23,87 & 16,81 & 18,60 & 40,72 \\
\hline Ni-Sn 1:1 & 14,97 & 23,43 & 24,70 & 36,89 \\
\hline
\end{tabular}

\section{.IV. CONCLUSÕES}

Os resultados mostraram que os catalisadores foram ativos e estáveis para a reação de hidrólise, uma vez que a reação não ocorre na ausência de catalisador. Foi verificado em todas as reações que ocorreu a conversão de glicerol por APR e a subsequente reação, a hidrogenação dos AGL insaturados produzidos a partir da hidrólise do TG. A temperatura de calcinação promoveu poucas mudanças nas propriedades dos catalisadores para a reação. No entanto, a adição de estanho aumentou a conversão de TG, diminuiu o rendimento em mono e di-glicerídeos e aumentou o rendimento em ácidos graxos livres. O rendimento e a seletividade em ácido esteárico também foram maiores no teste com o catalisador Ni-Sn. No entanto, o ácido linoleico foi o produto mais seletivo em todas as reações.

\section{REFERÊNCIAS}

CHENARD, G. D.; SALAZAR R.P.; OM N.T.; ARANDA D.G.A.; ALMARALES A.A., Hydrolysis-Hydrogenation of soybean oil and tallow. Natural Science,Vol.3, No.7, p. 530-534, 2011.

KING, J. W.; HOLLIDAY, R. L.; LIST, G. R. Hydrolysis of Soybean Oil in a Subcritical Water Flow Reactor. Green Chem. 1, p. 261-264, 1999.

MORETTO, E.; FETT, R., Tecnologia de óleos de gorduras vegetais na indústria de alimentos. São Paulo, Varela, V.150, 1998.

SCHEFFER B., MOLHOEK P., MOULIJN J.A., Appl. Catal. 46, p.11, 1989.

WANG, W. W; THAPALYA, N; CAMPOS, A; ROBERTS, W, L. Hydrogen fuels from vegetable oils vira hydrolisys and termo-catalytic descarbosxilation. Fuel, p. 622-629, 2012. 